\title{
Multimodality information fusion for aging pipe strength and toughness estimation using Bayesian networks
}

\author{
Jie Chen, Yongming Liu \\ Arizona State University, Tempe, $A Z$ \\ jchen378@asu.edu \\ yongming.liu@asu.edu
}

\begin{abstract}
Accurate estimation of the mechanical property of aging pipes is critical to maintain the safety and to scheduling maintenance. Destructive testing for mechanical properties measurement is very expensive and sometime impossible. Inference methods are needed for estimating the bulk properties by multimodality surface material measurements from nondestructive testing, such as chemical composition, volume fraction and hardness. Bayesian network modeling is utilized to integrate the information from various types of surface measurements for a more accurate bulk mechanical property estimation. To improve the approximation of the actual underlying model and avoid the risk of overfitting, Bayesian model averaging (BMA) of Bayesian networks is implemented to account for Bayesian network model uncertainty. The models considered are weighted based on the posterior model probability. Markov Chain Monte Carlo sampling provides an effective way for numerically computing the marginal likelihoods, which are essential for obtaining the posterior model probabilities. The predictive performance of single best model and BMA are compared by logarithmic scoring rule. The predictive capability of the proposed method is evaluated. It is shown that the Bayesian network and model averaging approach can provide more reliable results in predicting the bulk mechanical properties of the pipelines.
\end{abstract}

\section{PROBLEM STATEMENT}

The United States has become one of the leading nations that constructs the most millage length in the gas pipelines. Accurate pipe material mechanical property estimation is critical for the integrity and risk assessment of aging pipeline infrastructure systems. The objective of this research is to carry out Bayesian network-based data analytics approach to estimate the pipe material strength and toughness. Multimodality surface information are to be fused for the inference of the bulk properties. These surface measurements include surface chemistry, surface indentation and scratch testing results and microstructure

\footnotetext{
Jie Chen et al. This is an open-access article distributed under the terms of the Creative Commons Attribution 3.0 United States License, which permits unrestricted use, distribution, and reproduction in any medium, provided the original author and source are credited.
}

observations. A complete data set from 20 aging pips is available from industry and shown schematically in Figure 1. Each type of measurement contains certain amount of valuable information for pipe mechanical property estimation. Also, they are all associated with different amount of uncertainties. This research works on integrating all types of information in a statistical meaningful way for more reliable estimation.

Figure 1. Data set.

\section{EXPECTED CONTRIBUTIONS}

This research investigates the inference of the bulk mechanical properties of aging pipe materials using measured multimodality surface measurements. To achieve this goal, the methodology involves using the BMA of Bayesian networks as a general information fusion framework to incorporate the multimodal measurements and to derive the statistical inference. As a statistic based approach, Bayesian network has the capacity to improve robustness in modeling and reasoning under uncertainty. However, the number of high-scoring models is usually large especially when the given dataset has small sample size and several Bayesian networks describing the sparse training data equally well (Xiao, Chen, \& Chang, 2014). The single best Bayesian network structure does not always give a good approximation of the actual underlying structure (Kyu-Baek \& Byoung-Tak, 2005). The Bayesian model averaging (BMA) of Bayesian networks is utilized to enhance the confidence for decision making by considering the model associated uncertainties and model choice uncertainty (Guan, Jha, \& Liu, 2011).

For implementing BMA theory for Bayesian network model averaging, there exist the following difficulties. 1 . To 
calculate the model probability, the marginal likelihood of a model is needed whose closed form integral may not available. Markov Chain Monte Carlo (MCMC) approach provides a way to overcome this problem. 2. The number of models considered to be averaged may be extremely large. One approach is to reduce the number of models by leaving out the models which are not evidently supported by the data. This can be accomplished by introducing Occam's window to the process of BMA (Madigan \& Raftery, 1994). 3. A scoring metric is needed for assessing the predictive performance of BMA and the single models. The logarithmic scoring rule measures the predictive ability by comparing the probabilities each model assigns to the events that actually occur (Hoeting, Madigan, Raftery, \& Volinsky, 1999). This research focus on addressing the above issues to fulfill BMA of Bayesian networks for bulk mechanical estimation using multimodal surface data.

\section{RESEARCH PLAN}

To implement the Bayesian model averaging of the Bayesian networks, the research is carried out following two main steps. First, the models are simplified as naïve Bayesian network by assuming the surface measurements are independent given the bulk properties. Next, the Bayesian network are constructed by allowing the modeling of inter-feature-node dependencies to improve the quality of the inference process.

\subsection{Work Performed}

The first main step of the research plan is performed currently, i.e. BMA over the naïve Bayesian networks. Suppose there is a set of reasonable models $M_{1}, \ldots, M_{k}$ for estimating a quantity of interest $\mu$ from the set of data $y$. The marginal likelihood $\lambda_{n, j}(y)$ for model $M_{j}$ is expressed as

$$
\lambda_{n, j}(y)=\int f\left(y \mid M_{j}, \theta_{j}\right) \pi\left(\theta_{j} \mid M_{j}\right) d \theta_{j},
$$

where $f\left(y \mid M_{j}, \theta_{j}\right)$ is the likelihood of data given the model $M_{j}$ and its parameters $\theta_{j}, \pi\left(\theta_{j} \mid M_{j}\right)$ is the prior densities for

Table 1. Model ranks and posterior probabilities for the full data.

\begin{tabular}{cccccccccc}
\hline Model rank & $\mathrm{P}$ & $\mathrm{Cr}$ & $\mathrm{Cu}$ & $\mathrm{Si}$ & $\mathrm{GS}-1 / 2$ & $\mathrm{HD}$ & $\mathrm{VF}$ & $P\left(M_{j} \mid y\right)$ & $\begin{array}{c}\mathrm{BIC} \\
\text { rank }\end{array}$ \\
\hline 1 & & & $\bullet$ & & & $\bullet$ & & 0.169 & 1 \\
2 & & $\bullet$ & $\bullet$ & $\bullet$ & & $\bullet$ & $\bullet$ & 0.056 & 30 \\
3 & & $\bullet$ & $\bullet$ & $\bullet$ & $\bullet$ & $\bullet$ & & 0.053 & 34 \\
4 & & $\bullet$ & $\bullet$ & & $\bullet$ & $\bullet$ & $\bullet$ & 0.052 & 35 \\
5 & & $\bullet$ & & $\bullet$ & $\bullet$ & $\bullet$ & $\bullet$ & 0.049 & 40 \\
\hline \hline $\operatorname{Prall}\left(A_{i \neq 0}\right)$ & 0.34 & 0.66 & 0.83 & 0.42 & 0.33 & 0.84 & 0.39 & & \\
Proccam $\left(A_{i \neq 0}\right)$ & 0.37 & 0.62 & 0.77 & 0.44 & 0.36 & 0.78 & 0.41 & & \\
\hline
\end{tabular}

The performance of BMA for predictions of pipe strength is assessed by randomly splitting the data from a total of 20 aging pipes into two sets: build data (15

pipes) and test data (5 pipes). BMA shows better predictive performance than the best model. The log score of BMA is 0.4 less than that of the best. There are parameters $\theta_{j}$ of model $M_{j}$. The posterior probability of the model is obtained as

$$
P\left(M_{j} \mid y\right)=\frac{P\left(M_{j}\right) \lambda_{n, j}(y)}{\sum_{j^{\prime}=1}^{k} P\left(M_{j^{\prime}}\right) \lambda_{n, j^{\prime}}(y)},
$$

where $P\left(M_{j}\right)$ is prior probabilities for models under consideration. After computing the posterior density $\pi(\mu)$ $M j, y)$ of $\mu$ for each model, the averaged posterior distributions weighted by posterior model probability is given by

$$
\pi(\mu \mid y)=\sum_{j=1}^{k} P\left(M_{j} \mid y\right) \pi\left(\mu \mid M_{j}, y\right) .
$$

Eq. (3) shows that the posterior density $\pi(\mu \mid y)$ is a weighted average and mixture of the conditional posterior densities, where the weights are the posterior probability of each model. The posterior density $\pi(\mu \mid y)$ for the model averaging is the mixture distribution of the single conditional posterior density $\pi\left(\mu \mid M_{j}, y\right)$ considered.

The full data of 20 aging pipes are used for the Bayesian model selection, averaging and variable analysis. The results are shown in Table $1 . \operatorname{Pr}\left(A_{i \neq 0}\right)$ is the probability that the coefficient $A_{i}$ of a variable is non-zero and is used as a metric of evaluating the usefulness of a variable. The subscripts all and Occam show that the model set considered are the class of all models and the models selected by Occam's window, respectively. From the bottom row of Table $1, \operatorname{Pr}_{\text {all }}\left(A_{i \neq 0}\right)$ is close to Proccam $\left(A_{i \neq 0}\right)$, which means that Occam's window method which considers part of the models has similar results as that from the whole model set. It can be observed that the variables of best model $(\mathrm{Cu}$ and HD) have the highest probabilities that their coefficients are non-zero and show relatively strong predictive usefulness. The results provide positive evidence for $\mathrm{Cr}$ and indecisive evidence for P, Si, GS-1/2, and VF.
5 data in the test data set. Therefore the predictive probability of what is actually observed is $[\exp (0.4 / 5)-$ 
1] $\times 100 \%=8.3 \%$ larger for BMA than for best model

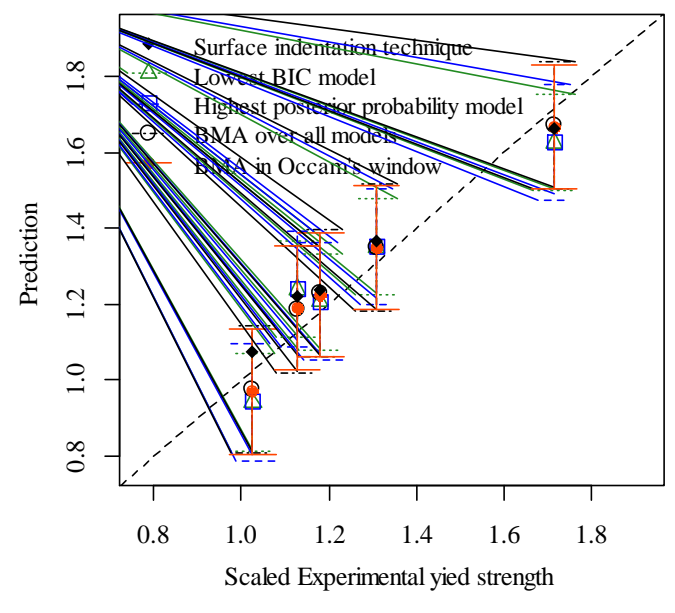

$\mathrm{M}_{1}$

Figure 2 shows intuitively the comparison of predictive performances of the direct surface indentation technique, lowest BIC model, highest posterior probability model, and BMA. The error bars represent the $95 \%$ credible intervals. The dots with different shapes are the expectations of different models. On average, the error of each model is less than that of the surface indentation technique. BMA shows better performance than the lowest BIC model and highest posterior probability model. In order to check that the above results are not due to the particular split used, the full data are randomly split for 100 times and the average results are analyzed. Among the repeated experiments of 100 times, BMA have better predictive ability for 71 times. The average predictive $\log$ sores are 15.07 and 14.26 for the highest posterior probability model and BMA, respectively. On average BMA can predict the strength with an improvement of $17.6 \%$.

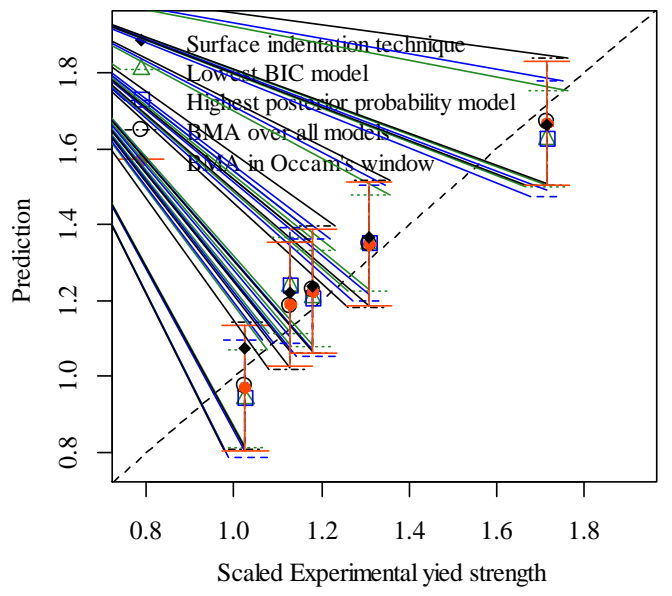

Figure 2. Predictive performance comparison.

\subsection{Remaining Work}

Naïve Bayesian network needs a strong assumption that the feature nodes (surface measurements) are independent from each other given the root node (bulk mechanical property), which is not always the case in practice. By considering the dependencies of the feature nodes, the quality of inference can be improved (Dash \& Cooper, 2004). The future work will focus on BMA over Bayesian networks with inter-feature-node dependences.

The remaining work includes:

1. Choosing the class of model over which to average needs an efficient algorithm for learning the top Bayesian network structures. Posterior model probabilities are used as a metric to guide the search. Occam's window has shown effectiveness in naïve Bayes case and will be extended for Bayesian networks.

2. Bayesian model averaging over the representative Bayesian networks. An appropriate method for averaging needs to be selected to perform the BMA efficiently.

3. Evaluation of the performance of BMA over Bayesian networks using the pipe data. The logarithmic scoring rule is to be used to measure how well a model predict future observations.

\section{CONCLUSION}

This research aims to estimate the bulk mechanical properties by fusing the multimodal surface measurements. To fulfill this objective, Bayesian model averaging of Bayesian networks is proposed for the implementation. The top models are selected and averaged according to the posterior model probability. The decision is made by averaging over the prediction results of the top Bayesian networks. BMA over naïve Bayesian networks has been performed and shows the BMA can improve the reliability and accuracy of the predictions. The remaining work will focus on the BMA considering the dependencies of the feature nodes to further improve the predictive capacity.

\section{REFERENCES}

Dash, D., \& Cooper, G. F. (2004). Model averaging for prediction with discrete Bayesian networks. Journal of Machine Learning Research, 5, 1177-1203.

Guan, X., Jha, R., \& Liu, Y. (2011). Model selection, updating, and averaging for probabilistic fatigue damage prognosis. Structural Safety, 33(3),

242-249. doi:10.1016/j.strusafe.2011.03.006

Hoeting, J. A., Madigan, D., Raftery, A. E., \& Volinsky, C. T. (1999). Bayesian Model 
Averaging: A Tutorial. Statistical Science, 14(4), 382-401.

Kyu-Baek, H., \& Byoung-Tak, Z. (2005). Bayesian model averaging of Bayesian network classifiers over multiple node-orders: application to sparse datasets. IEEE Transactions on Systems, Man, and Cybernetics, Part B (Cybernetics), 35(6), 1302-1310. doi:10.1109/TSMCB.2005.850162

Madigan, D., \& Raftery, A. E. (1994). Model Selection and Accounting for Model Uncertainty in Graphical Models Using Occam's Window. Journal of the American Statistical Association, $\quad$ 89(428), $\quad$ 1535-1546. doi:10.2307/2291017

Xiao, L., Chen, Y., \& Chang, C. K. (2014, 21-25 July 2014). Bayesian Model Averaging of Bayesian Network Classifiers for Intrusion Detection. Paper presented at the 2014 IEEE 38th International Computer Software and Applications Conference Workshops. 